\title{
Mobility Measurement in Nanowires Based on Magnetic Field-Induced Current Splitting Method in H-Shape Devices
}

\author{
Lucian Barbut, Farzan Jazaeri, Didier Bouvet, and Jean-Michel Sallese
}

\begin{abstract}
This work investigates a new method to measure mobility in nanowires. Based on a simple analytical approach and numerical simulations, we bring evidence that the traditional technique of Hall voltage measurement in low dimensional structures such as nanowires may generate large errors, while being challenging from a technological aspect. Here, we propose to extract the drift mobility in nanowires by measuring a variation of the electric current due to the presence of a magnetic field, in a specific nanowire network topology. This method overcomes the limitations inherent to the standard Hall effect technique and might open the way to a more precise and simple measurement of mobility in nanowires, still a matter of intensive research.
\end{abstract}

Index Terms-Hall effect, nanowire, current sensing, current splitting, carrier density, mobility measurement.

\section{INTRODUCTION}

$\mathbf{S}$ EMICONDUCTOR nanowires are of great interest nowadays given their potential use in electronic devices for integrated circuits, photovoltaics, biosensors etc. Because of their geometry, in many cases the intrinsic transport properties of nanowires such as carrier density, $n$, and mobility, $\mu$, are difficult to measure precisely.

In semiconductor industry the Y-function [1] has been widely used as simple method for MOSFET characterization allowing the estimation of the low field mobility in inversion mode transistor channel by measuring the drain current and the transconductance. However, as such, this approach is not suitable for depletion mode devices such as junctionless FETs [2], [3]. On the other hand, the $\mathrm{C}-\mathrm{V}$ measurement is also an interesting technique to estimate the carrier mobility of a semiconductor material. However, besides requiring dedicated MOSFET-like devices, when the dimensions are very small, this technique becomes inappropriate due to the very small capacitance to be measured contrasted by large parasitic contributions [4]-[7].

Another technique for $n$ and $\mu$ extraction is based on the Hall effect and consists in measuring the Hall voltage that builds up when a current is deviated by the Lorentz force. This is shown on Fig. 1. The presence of a magnetic field generates a Hall voltage $V_{\mathrm{H}}$ between two opposite edges of

Manuscript received January 14, 2014; accepted April 25, 2014.

This work was supported by the Swiss National Science Foundation organization (SNF), project 200020149822.

L. Barbut, F. Jazaeri, D. Bouvet, and J.-M. Sallese are with the Swiss Federal Institute of Technology in Lausanne (EPFL), 1015-Lausanne, Switzerland (phone: +41 2169 34602; e-mail: jean-michel.sallese@epfl.ch).

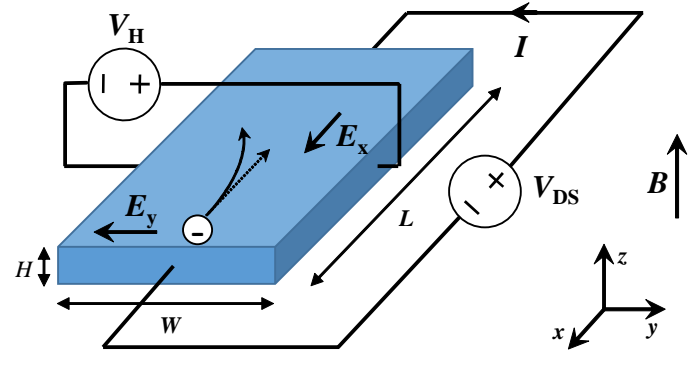

Fig. 1. Schematic view of a Hall Voltage measurement

the device when a current flows through it. In long devices $(L \gg W)$ the Hall voltage is expressed as [8]:

$$
V_{\mathrm{H}}=\frac{I B}{n e H} \text {. }
$$

The technique is rather generic and is commonly used on bulk or large scale structures. Because of geometrical and dimensional constrains, measuring the induced Hall voltage in nanowires is in fact very challenging. Lately, few research groups demonstrated for the first time such measurements in bottom-up fabricated nanowires [9], [10]. In both cases, the technique needs advanced e-beam lithography to achieve very precise alignment for the Hall contacts on the nanowire sidewalls. Particularly, the electrodes should to be placed perfectly in contact on the sidewalls of the nanowire, without short circuiting them and avoiding to cover the top of the nanowire. Another method has been proposed recently [11] which does not need to place Hall sensing electrodes exclusively on the sidewalls, but rather to set them at a distance of few tens of $\mathrm{nm}$ one from the other. The top of the nanowire is covered with an insulating material before the two evaporations, one for each Hall voltage electrode. However, the longitudinal offset that separates electrodes is a critical parameter as such, as the offset has to be as small as possible not to affect the sensitivity of the measurements, fact that still implies complex alignment of the e-beam lithographic steps.

We found however that, no matter the topology, additional electrodes integrated on the NW sidewalls may influence and drastically perturb the Hall voltage measurement. In fact, having Hall electrodes of a different material aside the nanowire can affect the distribution of the Hall-induced electric field when these have conductivities different than the "active region" under evaluation. 
In Section II, we are going to investigate this fact by carrying out a simple physics-based analysis and some simulations. Furthermore, in the Section III, we are introducing an alternative method for measuring the mobility in nanowires. Based on the current splitting in an H-shape device under magnetic field, we show how our method eliminates the fundamental problem described in Section II. A secondary but interesting feature of this method is the possibility to find the mobility without knowing the doping concentration, but simply by measuring the currents at the device terminals. An analytical model confirmed by TCAD simulations is also developed. Finally, in the last section we propose an abacuslike calculator that allows a direct evaluation of the mobility.

\section{Shortcomings of Classical Hall VOltage MEASUREMENT IN NANOWIRES}

\section{A. An analytical approach}

Applying the Hall effect principle, the Hall voltage $V_{\mathrm{H}}$ is extracted as the potential difference between the extremities of the Hall electrodes (Fig. 1). We can write (1) as function of the carrier mobility:

$$
V_{\mathrm{H}}=\frac{\mu B}{\sigma} I,
$$

where the link between the resistivity and mobility is

$$
\sigma=n e \mu .
$$

These two formulas are valid with some specific assumptions. First, the device is long enough $(L / W>5)$ and the Hall voltage probes are very small (compared to $L$ and $W$ ). Secondly, the electro-kinetic properties of the material must not be changed by the presence of a magnetic field. [8].

In Fig. 2 we reproduce a more realistic scheme of a nanowire device on which we want to measure the carrier mobility by using the Hall effect technique. The magnetic field is considered normal to the $x 0 y$ plane. We assume a uniformly doped $n$-type layer with a doping density $N_{\mathrm{D}}$ and thickness $H$. The current and its density $J$ are normalized by $H$, therefore measured in $\mathrm{A} / \mathrm{m}$ and $\mathrm{A} / \mathrm{m}^{-2}$, respectively. Assuming full ionization of donors and majority carrier density much higher that the intrinsic one, we can consider $n=N_{\mathrm{D}}$. Note that we will rely on these assumptions for all analysis throughout this work.

We will carry out a deeper analysis, considering also the change in the kinetic properties due to the presence of the magnetic field. While still being able to consider it a long device, the Hall electrodes $\left(V_{\mathrm{H}+}\right.$ and $V_{\mathrm{H}-}$ above and under the nanowire, in blue) are not negligible in size, compared to the nanowire itself (in red). The current density in any point of the device is:

$$
\boldsymbol{J}=\sigma_{\mathrm{B}} \boldsymbol{E}+\sigma_{\mathrm{B}} \mu_{\mathrm{H}} \boldsymbol{E} \times \boldsymbol{B},
$$

where

$$
\mu_{\mathrm{H}}=-r_{\mathrm{H}} \mu
$$

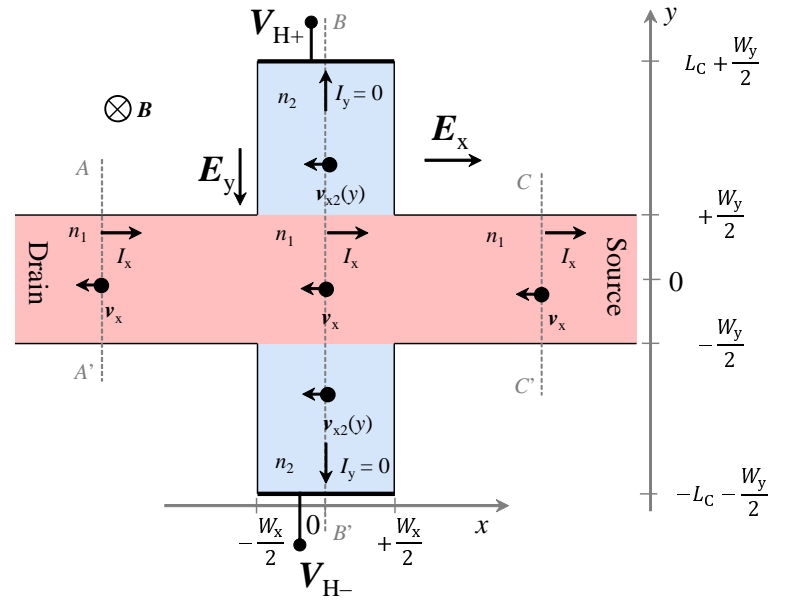

Fig. 2. Top view of an extremely downscaled device for Hall effect measurement. The Hall voltage electrodes (in blue) have the width similar to the one of the nanowire under measurement (in red).

is the Hall mobility ((3.211) in [8]) and

$$
\sigma_{\mathrm{B}}=\sigma\left(1-1.77 \mu^{2} B^{2}\right)
$$

is the effective conductivity of silicon ((3.269) in [8]), when taking into account the geometrical and electrical magnetoresistance effects. The coefficient $r_{\mathrm{H}}$ is the Hall factor, considered constant and equal to 1.15 in this work [12], [13], while $\sigma$ and $\mu$ are the conductivity and drift mobility, as in the approximation (2). Although part of the current injected in the device can spread inside the Hall electrodes, no current flows in/out of them except through the source/drain contacts: $I_{\mathrm{y}}=0$. This condition can be formalized as:

$$
\int_{-\frac{W_{\mathrm{x}}}{2}}^{\frac{W_{\mathrm{x}}}{2}} J_{\mathrm{y}} d x=0=\int_{-\frac{W_{\mathrm{x}}}{2}}^{\frac{W_{\mathrm{x}}}{2}} \sigma_{\mathrm{B}} E_{\mathrm{y}} d x+B \int_{-\frac{W_{\mathrm{x}}}{2}}^{\frac{W_{\mathrm{x}}}{2}} \sigma_{\mathrm{B}} \mu_{\mathrm{H}} E_{\mathrm{x}} d x
$$

which further gives

$$
\int_{-\frac{W_{\mathrm{x}}}{2}}^{\frac{W_{\mathrm{x}}}{2}} E_{\mathrm{x}} d x=-\frac{1}{B} \int_{-\frac{W_{\mathrm{x}}}{2}}^{\frac{W_{\mathrm{x}}}{2}} \frac{E_{\mathrm{y}}}{\mu_{\mathrm{H}}} d x .
$$

Integrating the $x$-component of the current density in (4), on $y$-axis from $V_{\mathrm{H}-}$ and $V_{\mathrm{H}+}$ and along the $x$-axis for the Hall contacts width, we have:

$$
\begin{aligned}
& I_{\mathrm{x}} W_{\mathrm{x}}=\int_{-\frac{W_{\mathrm{x}}}{2}}^{\frac{W_{\mathrm{x}}}{2}} \int_{-L_{\mathrm{C}}-\frac{W_{\mathrm{y}}}{2}}^{L_{\mathrm{C}}+\frac{W_{\mathrm{y}}}{2}} J_{\mathrm{x}} d x d y= \\
& \sigma_{\mathrm{B}} \int_{-\frac{W_{\mathrm{x}}}{2}}^{\frac{W_{\mathrm{x}}}{2}} \int_{-L_{\mathrm{C}}-\frac{W_{\mathrm{y}}}{2}}^{L_{\mathrm{C}}+\frac{W_{\mathrm{y}}}{2}} E_{\mathrm{x}} d x d y+\sigma_{\mathrm{B}} \mu_{\mathrm{H}} B \int_{-\frac{W_{\mathrm{x}}}{2}}^{\frac{W_{\mathrm{x}}}{2}} \int_{-L_{\mathrm{C}}-\frac{W_{\mathrm{y}}}{2}}^{L_{\mathrm{C}}+\frac{W_{\mathrm{y}}}{2}} E_{\mathrm{y}} d x d y .
\end{aligned}
$$

We can extract the first member of the sum in (9) by integrating (8) along the BB' cross-section. Rewriting (9) only as function of $E_{\mathrm{y}}$, we obtain: 


$$
I_{\mathrm{x}} W_{\mathrm{x}}=\sigma_{\mathrm{B}} \frac{1-\mu_{\mathrm{H}}^{2} B^{2}}{-\mu_{\mathrm{H}} B} \int_{-\frac{W_{\mathrm{x}}}{2}}^{\frac{W_{\mathrm{x}}}{2}} \int_{-L_{\mathrm{C}}-\frac{W_{\mathrm{y}}}{2}}^{L_{\mathrm{C}}+\frac{W_{\mathrm{y}}}{2}} E_{\mathrm{y}} d x d y .
$$

In the same time, the integration of $E_{\mathrm{y}}$ along the crosssection $B B^{\prime}$ gives the Hall voltage between $V_{\mathrm{H}+}$ and $V_{\mathrm{H}-}$ contacts:

$$
V_{\mathrm{H}}=\int_{-L_{\mathrm{C}}-W_{\mathrm{y}} / 2}^{L_{\mathrm{C}}+W_{\mathrm{y}} / 2} E_{\mathrm{y}} d y
$$

which can be considered constant along the contact width $W_{\mathrm{x}}$. Therefore, using (10) and (11), we find the link between the current passing through the device and the Hall potential between $V_{\mathrm{H}+}$ and $V_{\mathrm{H}-}$ :

$$
V_{\mathrm{H}}=\frac{-\mu_{\mathrm{H}} B}{\sigma_{\mathrm{B}}\left(1-\mu_{\mathrm{H}}^{2} B^{2}\right)} I_{\mathrm{x}},
$$

which is equivalent to (2), additionally taking into account the material response to the magnetic field. This last relation is valid only when the nanowire and the Hall electrodes share the same kinetic properties $\left(n_{1}=n_{2}=n\right)$, as $\sigma_{\mathrm{B}}$ and $\mu_{\mathrm{H}}$ will be the same in all the regions along BB'. In this case, there is no impact of the Hall electrodes on the measured Hall voltage, fact that is usually accepted.

On the other hand, let us assume that the carrier density in the Hall probe extensions can be different (the blue regions in Fig. 2, $n_{2}$ ) from the one in the nanowire (Fig. 2, $n_{1}$ ), which is indeed the case in [10] and [11]. The Hall mobility $\mu_{\mathrm{H}}$ and the effective resistivity $\sigma_{\mathrm{B}}$ are different for each region, therefore relations (9), (10) and (12) are not valid anymore. The current $I_{\mathrm{x}}$ needs to be expressed as sum of the contributions relative to the three distinct regions: from $-L_{\mathrm{C}}-W_{\mathrm{y}} / 2$ to $-W_{\mathrm{y}} / 2$, from $-W_{\mathrm{y}} / 2$ to $W_{\mathrm{y}} / 2$ and from $W_{\mathrm{y}} / 2$ to $L_{\mathrm{C}}+W_{\mathrm{y}} / 2$. For the sake of simplicity, we consider contributions to $I_{\mathrm{x}}$ from the first and the third regions as equal.

Relation (10) becomes

$$
\begin{aligned}
I_{\mathrm{x}} W_{\mathrm{x}} & =\sigma_{\mathrm{B} 1} \frac{1-\mu_{\mathrm{H} 1}^{2} B^{2}}{-\mu_{\mathrm{H} 1} B} \int_{-\frac{W_{\mathrm{x}}}{2}}^{\frac{W_{\mathrm{x}}}{2}} \int_{-\frac{W_{\mathrm{y}}}{2}}^{\frac{W_{\mathrm{y}}}{2}} E_{\mathrm{y}} d x d y+ \\
& +2 \sigma_{\mathrm{B} 2} \frac{1-\mu_{\mathrm{H} 2}^{2} B^{2}}{-\mu_{\mathrm{H} 2} B} \int_{-\frac{W_{\mathrm{x}}}{2}}^{\frac{W_{\mathrm{x}}}{2}} \int_{\frac{W_{\mathrm{y}}}{2}}^{L_{\mathrm{C}}+\frac{W_{\mathrm{y}}}{2}} E_{\mathrm{y}} d x d y .
\end{aligned}
$$

Without reproducing all the steps, from (11) and (13) we find

$$
\begin{aligned}
& V_{\mathrm{H}}=\frac{-\mu_{\mathrm{H} 1} B}{\sigma_{\mathrm{B} 1}\left(1-\mu_{\mathrm{H} 1}^{2} B^{2}\right)} I_{\mathrm{x}}+ \\
& +\frac{2 \sigma_{\mathrm{B} 2}}{W_{\mathrm{x}}}\left(1-\frac{\sigma_{\mathrm{B} 2} \mu_{\mathrm{H} 1}\left(1-\mu_{\mathrm{H} 2}^{2} B^{2}\right)}{\sigma_{\mathrm{B} 1} \mu_{\mathrm{H} 2}\left(1-\mu_{\mathrm{H} 1}^{2} B^{2}\right)}\right) \int_{-\frac{W_{\mathrm{x}}}{2}}^{\frac{W_{\mathrm{x}}}{2}} \int_{\frac{W_{\mathrm{y}}}{2}}^{L_{\mathrm{C}}+\frac{W_{\mathrm{y}}}{2}} E_{\mathrm{y}} d x d y .
\end{aligned}
$$

Comparing (12) with (14), we notice an extra term, which accounts for the "difference" between the two materials. In order to make the explanation more clear, we assume that

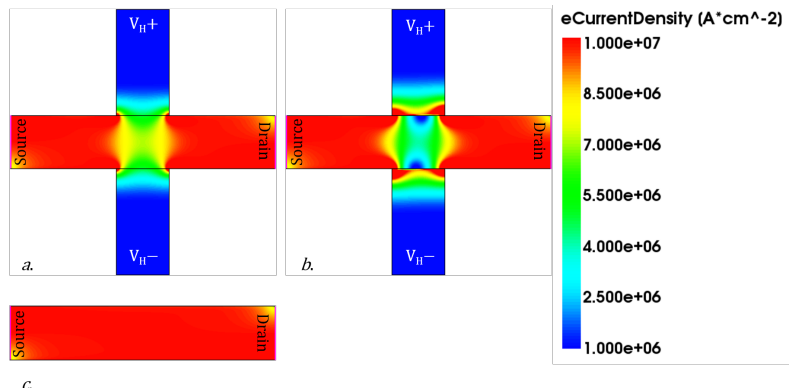

Fig. 3. Two-dimensional TCAD simulation showing current density distribution in a nanowire (from source to drain) for detection of Hall voltage. In $a$., the device is uniformly doped $n$-type $N_{\mathrm{D}}=1 \times 10^{19} \mathrm{~cm}^{-3}$ in both nanowire and $V_{\mathrm{H}}$ contacts. In $b$. the electrodes are degenerated $\left(1 \times 10^{21} \mathrm{~cm}^{-3}\right)$, acting similarly to a metal. In $c$. the NW is doped $N_{\mathrm{D}}=1 \times 10^{19} \mathrm{~cm}^{-3}$ and there are no Hall electrodes. The nanowire is $250 \mathrm{~nm}$ length and $50 \mathrm{~nm}$ width, and each Hall sensing contact is $50 \mathrm{~nm}$ width $\left(W_{\mathrm{x}}\right)$ and $100 \mathrm{~nm}$ length $\left(L_{\mathrm{C}}\right)$. For all cases, $V_{\mathrm{DS}}=0.1 \mathrm{~V}$ and $r_{\mathrm{H}} B=1.15 \mathrm{~T}$.

$\mu_{\mathrm{H}}^{2} B^{2} \ll 1$ for both materials and the scattering factors $r_{\mathrm{H} 1}=$ $r_{\mathrm{H} 2} \approx 1$. With this simplification, (14) becomes:

$$
V_{\mathrm{H}}=\frac{B I_{\mathrm{x}}}{n_{1} e}+\frac{2}{W_{\mathrm{x}}}\left(1-\frac{n_{2}}{n_{1}}\right) \int_{-\frac{W_{\mathrm{x}}}{2}}^{\frac{W_{\mathrm{x}}}{2}} \int_{\frac{W_{\mathrm{y}}}{2}}^{L_{\mathrm{C}}+\frac{W_{\mathrm{y}}}{2}} E_{\mathrm{y}} d x d y .
$$

We note that the ratio $n_{2} / n_{1}$ is playing an important role in the second term of the sum, given that it can easily reach values of the order of hundreds. Thus it becomes evident that we cannot relate the Hall voltage $V_{\mathrm{H}}$ to the current $I_{\mathrm{x}}$ ignoring the influence of the Hall electrodes, both from geometrical $\left(L_{\mathrm{C}}\right.$ and $\left.W_{\mathrm{x}}\right)$ and electrical points of view. This is a major point.

Our analysis suggests therefore that the Hall voltage might strongly depart from the ideal case when the channel and the probes share different doping, as it should also include non negligible contributions from the access nodes. This is particularly true when the device of interest reaches dimensions of tens or hundreds of nanometers. In the following section, we propose to quantify this analysis by numerical simulations.

\section{B. Numerical analysis}

In order to verify the predictions of the analytical approach, we performed two dimensional TCAD simulations, using Synopsys Sentaurus (v. 2012.06), on a nanowire device as detailed in Fig. 2, with few different scenarios concerning the Hall electrodes:

a. electrodes doped as the central nanowire,

$b$. electrodes doped two orders of magnitude higher than for the nanowire (degenerated semiconductor, equivalent to metal electrodes),

c. no Hall electrodes.

Note that scenario $c$. should represent the ideal situation, where the nanowire is completely free of influences that might alter the Hall voltage extraction, but unfeasible from a practical point of view.

Fig. 3 shows a comparison of the simulation results of the three scenarios described above. Since the current distribution 
TABLE I

HALL VOLTAGE EXTRACTED FROM TCAD SIMULATION AND RELATIVE CALCULATION OF CARRIER DENSITY AND MOBILITY FOR THE DEVICES AS IN FIG. 3, WITH THE NANOWIRE UNIFORMLY DOPED $N_{\mathrm{D}}=1 \times 10^{19}$, $\mu=500 \mathrm{~cm}^{2} \mathrm{~V}^{-1} \mathrm{~s}^{-1}, V_{\mathrm{DS}}=0.1 \mathrm{~V}, r_{\mathrm{H}} B=1.15 \mathrm{~T}$.

\begin{tabular}{c|c|c|c|c} 
Contacts type & $\begin{array}{c}V_{\mathrm{H}} \\
(\mathrm{mV})\end{array}$ & $\begin{array}{c}I_{\mathrm{DS}} \\
(\mathrm{mA} / \mu \mathrm{m})\end{array}$ & $\begin{array}{c}n \\
\left(\mathrm{~cm}^{-3}\right)\end{array}$ & $\begin{array}{c}\mu \\
\left(\mathrm{cm}^{2} /(\mathrm{Vs})\right)\end{array}$ \\
\hline no contacts at all & 1.204 & 1.765 & $1.05 \times 10^{19}$ & 475.0 \\
$\mathrm{Si}, N_{\mathrm{D}}=1 \times 10^{19}$ & 1.263 & 1.760 & $1.00 \times 10^{19}$ & 499.8 \\
$\mathrm{Si}, N_{\mathrm{D}}=1 \times 10^{20}$ & 0.743 & 1.762 & $1.70 \times 10^{19}$ & 293.7 \\
$\mathrm{Si}, N_{\mathrm{D}}=1 \times 10^{21}$ & 0.649 & 1.762 & $1.95 \times 10^{19}$ & 256.3 \\
ideal metal & 0.640 & 1.764 & $1.98 \times 10^{19}$ & 252.7
\end{tabular}

is affected by the presence of the sensing electrodes, one could expect that this would also modify the Hall induced electric field. In our study, we consider a device where the central part of the nanowire is doped $N_{\mathrm{D}}=1 \times 10^{19} \mathrm{~cm}^{-3}$ and the mobility is constant $\mu=500 \mathrm{~cm}^{2} \mathrm{~V}^{-1} \mathrm{~s}^{-1}$. Table I shows $N_{\mathrm{D}}$ and $\mu$ as extracted using the classical Hall effect measurement described in the previous section, for the three scenarios presented above. We have used (14), considering the approximation $\mu_{\mathrm{H}}^{2} B^{2} \ll 1$. (Note that if neglected, this would generate at maximum $2 \%$ of variation.)

Scenario $c$ is expected to give the most accurate value for the mobility and carrier density as it should not perturb the electrostatics of the system. Probing the Hall voltage at the nanowire/air interface, we find that the mobility and carrier density are well predicted, with value departing from the expected ones less than $5 \%$. Concerning case $b$. (with contacts doped $1 \times 10^{21} \mathrm{~cm}^{-3}$ ) the carrier density is overestimated by more than $90 \%$ and, more importantly, the drift mobility deviates by approximately $50 \%$. For electrodes having the electrical properties closer to the nanowire ones, we noticed that the mismatch is decreased (e.g. for contacts doped $1 \times 10^{20} \mathrm{~cm}^{-3}$, the mobility is about $40 \%$ lower than expected.) In case $a$., where the doping density in the Hall contacts is the same as in nanowire, simulations predict the most accurate values that differ less then $1 \%$ from the expected ones, thus confirming the analysis done previously.

Unexpectedly, the worst case happens for "ideal" metal electrodes (Table I). The analysis presented above leads to the conclusion that such an approach suffers from conceptual limitations which seem to have been overlooked until now. It results that, given the small dimensions of the device to be tested, access electrodes are influencing the Hall voltage in a non negligible amount, exception being made when they have the same doping as the core device.

Therefore, an alternative has to be found to measure $\mu$ in NW under magnetic field. Unlike this classical approach, we propose to measure the mobility by means of current measurements in a specific topology.

\section{CuRrent SPlitTing IN H-Shape NANOWire TOPOLOGY}

\section{A. Working principles}

The central idea of the mobility extraction principle that we propose is based upon current measurements in a symmetric

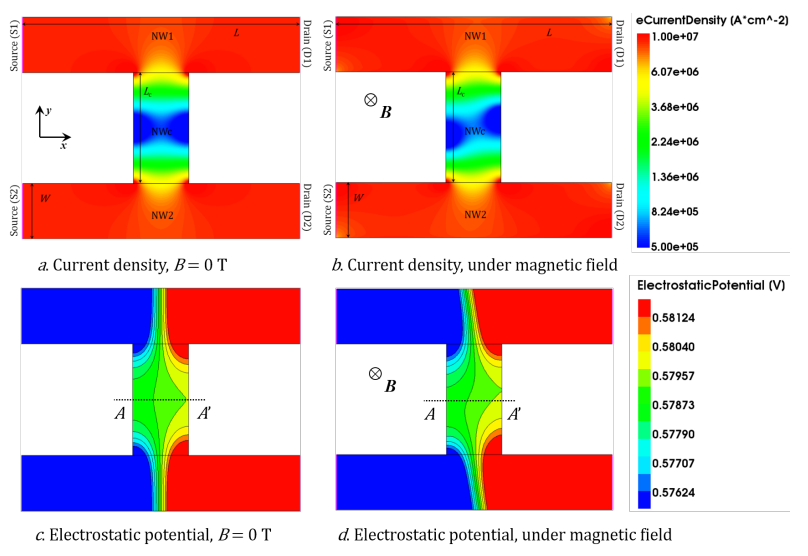

Fig. 4. Two-dimensional TCAD simulation with the change in current density and potential distribution in the H-shape device. $N_{\mathrm{D}}=1 \times 10^{19} \mathrm{~cm}^{-3}$, $V_{\mathrm{DS}}=0.1 \mathrm{~V}, r_{\mathrm{H}} B=1.15 \mathrm{~T}$. NW1 and NW2 are $250 \mathrm{~nm}$ length $(L)$ and $50 \mathrm{~nm}$ width $(W)$, and NWc $50 \mathrm{~nm}$ width $\left(W_{\mathrm{C}}\right)$ and $100 \mathrm{~nm}$ length $\left(L_{\mathrm{C}}\right)$.

H-shaped structure as presented in Fig. 4. The device has four independent terminals nodes named as sources (left-side nodes, S1 and S2) and drains (the right-side nodes D1 and D2) which are used as current probes. Basically, when no magnetic field is applied to the device, the system depicted in Fig. 4a is fully symmetric with respect to the cross-section $A A^{\prime}$. Therefore, if $V_{\mathrm{S} 1}=V_{\mathrm{S} 2}$ and $V_{\mathrm{D} 1}=V_{\mathrm{D} 2}$, we expect that the currents flowing in each of the nanowires NW1 and NW2 will be equal. It further implies that the net transfer of current through the central nanowire NWc is null. This trivial situation is confirmed by TCAD simulations where the potential distribution is fully symmetric across $A A^{\prime}$ (Fig. 4c) and where all currents are equal $\left(I_{\mathrm{S} 1}=I_{\mathrm{S} 2}=I_{\mathrm{D} 1}=I_{\mathrm{D} 2}\right)$.

Now we can wonder what happens when a magnetic field $B$ is applied normal to the surface (perpendicular to the $x 0 y$ plane). In this case, the symmetry across the axis $A A^{\prime}$ is lost, causing some current to flow between NW1 and NW2 through NWc. According to TCAD simulations, for the silicon nanowire device represented in Fig. $4 \mathrm{~b}$ doped at $1 \times 10^{19} \mathrm{~cm}^{-3}$, we obtain a current mismatch between $I_{\mathrm{S} 1}$ and $I_{\mathrm{D} 1}$ of about $2.6 \%\left(V_{\mathrm{DS}}=0.1 \mathrm{~V}\right.$ and $\left.\mu=1000 \mathrm{~cm}^{2} \mathrm{~V}^{-1} \mathrm{~s}^{-1}\right)$ under a magnetic field of $1 \mathrm{~T}$. As it will be detailed in next section, this is attributed to an excess current flowing through the central nanowire that accounts for electrons deflected by the magnetic field.

\section{B. The current splitting in H-shape device: an analytical approach}

In this section, we present a simple analytical model of the current splitting principle, having in mind that in this first attempt the objective is not so much to obtain a high accuracy, but rather to get an insight in predicting invariant laws that will finally be used to build some generic rules for mobility extraction.

A picture of the current splitting effect is illustrated on Fig. 5. The current imposed by the external source is always assumed to flow along the $x$-axis, in all the regions of the device (NW1, NW2 and NWc). 


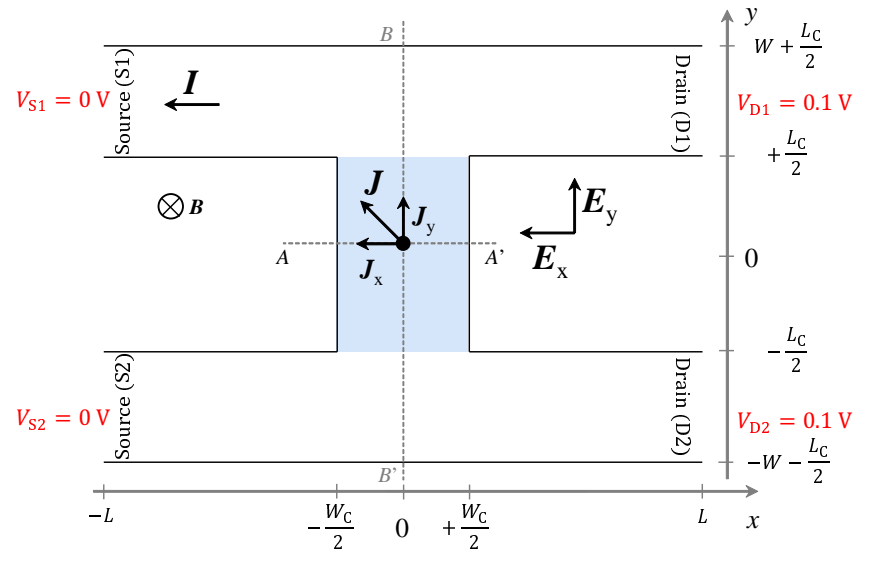

Fig. 5. Simple picture of a device as in Fig. 4, showing the currents and electric fields, under a normal magnetic field.

To better understand how current splitting arises in such $\mathrm{H}$ shape device, we will analyze an ideal case leading to simple but highly instructive analytical relationships.

As we did for the usual Hall voltage approach, we can treat the problem in two dimensions, assuming the magnetic field normal to $x_{0 y}$ plane [8]. Projecting (4) on $x$ - and $y$-axis we have the two scalar components of the current density, for any points in the device:

$$
J_{\mathrm{x}}=\sigma_{\mathrm{B}} E_{\mathrm{x}}+\sigma_{\mathrm{B}} \mu_{\mathrm{H}} E_{\mathrm{y}} B
$$

and

$$
J_{\mathrm{y}}=\sigma_{\mathrm{B}} E_{\mathrm{y}}+\sigma_{\mathrm{B}} \mu_{\mathrm{H}} E_{\mathrm{x}} B
$$

The full picture is relatively complex, as both $x$ - and $y$ components of the electric field are present in both relations. Therefore, throughout the following analysis we will assume some simplifications when needed, without harming the correctness of the demonstration.

The total current (in absolute value) passing through the device from source to drain can obtained by integrating $J_{\mathrm{x}}$ over the cross section $B B^{\prime}$ (or in any other one, parallel to it):

$$
I_{\mathrm{x}}=\int_{-W-\frac{L_{\mathrm{C}}}{2}}^{W+\frac{L_{\mathrm{C}}}{2}}\left(\sigma_{\mathrm{B}} E_{\mathrm{x}}+\sigma_{\mathrm{B}} \mu_{\mathrm{H}} E_{\mathrm{y}} B\right) d y=I_{\mathrm{S} 1}+I_{\mathrm{S} 2} .
$$

Since we aim at finding the excess current passing through the interconnection nanowire $\mathrm{NWc}$, we can evaluate the current through the cross-section $A A^{\prime}$ in Fig. 5:

$$
I_{\mathrm{y}}=\int_{-\frac{W_{\mathrm{C}}}{2}}^{\frac{W_{\mathrm{C}}}{2}} J_{\mathrm{y}} d x \text {. }
$$

From (16) and (17) and (19), we obtain

$$
I_{\mathrm{y}}=\int_{-\frac{W_{\mathrm{C}}}{2}}^{\frac{W_{\mathrm{C}}}{2}}\left[\sigma_{\mathrm{B}}\left(1-\mu_{\mathrm{H}}^{2} B^{2}\right) E_{\mathrm{y}}+\mu_{\mathrm{H}} B J_{\mathrm{x}}\right] d x .
$$

At this point, we can integrate on $y$-axis, along $B B^{\prime}$ :

$$
\begin{aligned}
I_{\mathrm{y}}\left(2 W+L_{\mathrm{C}}\right)= & \sigma_{\mathrm{B}}\left(1-\mu_{\mathrm{H}}^{2} B^{2}\right) \int_{-\frac{W_{\mathrm{C}}}{2}}^{\frac{W_{\mathrm{C}}}{2}} \int_{-W-\frac{L_{\mathrm{C}}}{2}}^{W+\frac{L_{\mathrm{C}}}{2}} E_{\mathrm{y}} d x d y+ \\
& +\mu_{\mathrm{H}} B \int_{-\frac{W_{\mathrm{C}}}{2}}^{\frac{W_{\mathrm{C}}}{2}} \int_{-W-\frac{L_{\mathrm{C}}}{2}}^{W+\frac{L_{\mathrm{C}}}{2}} J_{\mathrm{x}} d x d y .
\end{aligned}
$$

Note that the first double integral represents a potential drop, which we call $V_{\mathrm{H}}$, multiplied by $W_{\mathrm{C}}$, assuming that this potential drop is constant along $W_{\mathrm{C}}$. In the same time, the second integral in (21) can be easily expressed as function of $B$ and $I_{\mathrm{S} 1}+I_{\mathrm{S} 2}$, taking into account (18). Relation (21) can be rewritten as

$$
I_{\mathrm{y}}=\frac{W_{\mathrm{C}}}{2 W+L_{\mathrm{C}}}\left(\sigma_{\mathrm{B}}\left(1-\mu_{\mathrm{H}}^{2} B^{2}\right) V_{\mathrm{H}}+\mu_{\mathrm{H}} B I_{\mathrm{x}}\right) .
$$

As we consider weak magnetic induction, $(\mu B)^{2} \ll 1$, we can simplify (22). With (5), we get:

$$
I_{\mathrm{y}}=\frac{W_{\mathrm{C}}}{2 W+L_{\mathrm{C}}} n e \mu\left(V_{\mathrm{H}}-r_{\mathrm{H}} \frac{B\left(I_{\mathrm{S} 1}+I_{\mathrm{S} 2}\right)}{n e}\right) .
$$

Given that the second member of the sum containing $B$ is also a potential (which we call $V_{\mathrm{b}}$ ), this equation could be intended as Ohm's law

$$
I_{\mathrm{y}}=\frac{1}{R^{\prime}}\left(V_{\mathrm{H}}-V_{\mathrm{b}}\right),
$$

where $R^{\prime}$ is the equivalent resistance of the central part of the device, i.e., the region $\mathrm{NWc}$ plus the additional parts corresponding to its width (from NW1 and NW2):

$$
R^{\prime}=\frac{2 W+L_{\mathrm{C}}}{n e \mu W_{\mathrm{C}}} \text {. }
$$

Based on (24), an equivalent representation as an electric circuit of this device under magnetic field is proposed in Fig. 6. $V_{\mathrm{H}}=V_{\mathrm{B}}-V_{\mathrm{A}}$ is intended positive and approximates the potential difference between the extremities of the device along the cross-section $B B^{\prime}$. From the circuit we obtain $I_{\mathrm{y}}$ as function of the geometric parameters, including $B$ and $V_{\mathrm{D}}$. Solving the linear system of equations we find a fundamental link between the current in NWc and the device parameters:

$$
I_{\mathrm{y}}=\Delta I=\frac{r_{\mathrm{H}} B V_{\mathrm{D}}}{R\left(R+R^{\prime}\right) n e}=\frac{r_{\mathrm{H}} B V_{\mathrm{D}}}{\frac{L}{W}+\frac{2 W+L_{\mathrm{C}}}{W_{\mathrm{C}}}} \frac{W}{L} n e \mu^{2} .
$$

Relation (26) represents the variation of current between $\mathrm{S} 1$ and D1 nodes, i.e., $\Delta I=I_{\mathrm{S} 1}-I_{\mathrm{D} 1}$. Note that this current varies as the square of the mobility.

From the same equations, we can find $I_{\mathrm{S} 1}$ and $I_{\mathrm{D} 1}$ :

$$
\begin{aligned}
I_{\mathrm{S} 1 / \mathrm{D} 1} & =\frac{V_{\mathrm{D}}}{2 R}\left(1 \pm \frac{r_{\mathrm{H}} B}{R\left(R+R^{\prime}\right) n e}\right) \\
& =\frac{V_{\mathrm{D}} W n e \mu}{2 L}\left(1 \pm \frac{r_{\mathrm{H}} \mu B}{\frac{L}{W}+\frac{2 W+L_{\mathrm{C}}}{W_{\mathrm{C}}}}\right) .
\end{aligned}
$$

Finally, combining the last two equations, we find that the relative change in the source current upon the magnetic field obeys to a simple relationship:

$$
\frac{\Delta I}{I_{\mathrm{S} 1 / \mathrm{D} 1}}=\frac{2 r_{\mathrm{H}} \mu B}{\frac{L}{W}+\frac{2 W+L_{\mathrm{C}}}{W_{\mathrm{C}}} \pm r_{\mathrm{H}} \mu B} .
$$




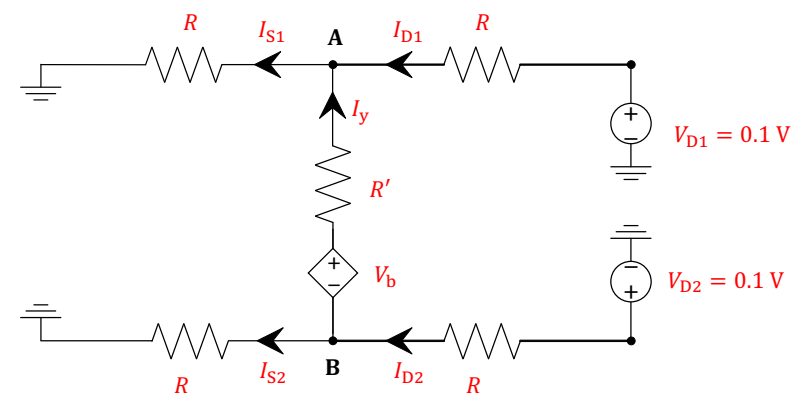

Fig. 6. Electric circuit equivalent for the device under study, based on (24).

This relation is the main result of this work as it simply links the mobility to the relative variation of the current in the $\mathrm{H}$ shape topology. While not being influenced by doping (carrier density $N_{\mathrm{D}}$ ) and by the absolute value of current in NW1 and NW2 (controlled by $V_{\mathrm{DS}}$ ), this ratio depends essentially on the mobility of the material and the geometrical dimensions. It is important to note that the term containing the mobility in the denominator plays a negligible role. Given the values of the other parameters and supposing $B=1 \mathrm{~T}$ and $\mu=$ $1000 \mathrm{~cm}^{2}(\mathrm{Vs})^{-1}$, in a rough approximation this term is at least 10 times smaller than the rest of the denominator. Therefore, we can affirm that the ratio $\Delta I / I$ is "almost" linear with $\mu$.

From (28), we can extract a relationship between the mobility in the central nanowire, NWc, the applied magnetic field, and the current measured at the device terminals:

$$
\mu=\frac{1}{r_{\mathrm{H}} B\left(2 \mp \frac{\Delta I}{I_{\mathrm{S} 1 / \mathrm{D} 1}}\right)} \frac{\Delta I}{I_{\mathrm{S} 1 / \mathrm{D} 1}}\left(\frac{L}{W}+\frac{2 W+L_{\mathrm{C}}}{W_{\mathrm{C}}}\right) .
$$

As noted in the case of (28), we could simplify (29) by neglecting the contribution of $\Delta I / I$ to the denominator, given that the error generated would be less than $2 \%$. As we will see in the following subsections, we still need to introduce some correction to (29) if we want it to be more accurate, since some assumptions regarding the potential drop $V_{\mathrm{H}}$ has been made for obtaining it. However, (29) is still of major interest as it predicts some key rules such as an invariance with respect to scaling. Any uniform scaling applied to $W_{\mathrm{C}}, L_{\mathrm{C}}, W$, and $L$ is expected to have no impact on $\mu$.

As an important feature, these invariant rules suggest the use of an abacus to get the mobility from current variations upon the magnetic field. In the next subsection, we will analyze this approach through extensive TCAD simulations and discuss the range of validity of (29).

\section{TCAD simulations and model assessment}

This section has two major objectives. The first one is to evaluate the invariance predicted by (29) with respect to the doping, scaling and biasing voltages, making use of numerical simulations. Additionally, we can also see how these relationships can be used as a first step for mobility estimation and eventually how we can introduce some correction to balance some former assumptions.

The second objective is to propose an abacus-like design space, based on the model as well as on TCAD simulations.
TABLE II

$\Delta I / I$ VERSUS MOBILITY FOR DIFFERENT DOPING, $V_{\mathrm{DS}}=0.1 \mathrm{~V}$

\begin{tabular}{c|c|c}
$\begin{array}{c}N_{\mathrm{D}} \\
\left(\mathrm{cm}^{-3}\right)\end{array}$ & $\begin{array}{c}\mu \\
\left(\mathrm{cm}^{2}(\mathrm{Vs})^{-1}\right)\end{array}$ & $\begin{array}{c}\Delta I / I_{\mathrm{S} 2} \\
(\%)\end{array}$ \\
\hline $5 \times 10^{17}$ & 250 & 0.64 \\
$5 \times 10^{17}$ & 500 & 1.28 \\
$5 \times 10^{17}$ & 1000 & 2.57 \\
\hline $2.5 \times 10^{18}$ & 250 & 0.62 \\
$2.5 \times 10^{18}$ & 500 & 1.26 \\
$2.5 \times 10^{18}$ & 1000 & 2.60 \\
\hline $1 \times 10^{19}$ & 250 & 0.62 \\
$1 \times 10^{19}$ & 500 & 1.25 \\
$1 \times 10^{19}$ & 1000 & 2.62
\end{tabular}

In such a way, for a given H-shape geometry, one will be able to predict the mobility in a nanowire by measuring the variation of the electric current upon magnetic field.

In order to better assess the validity of this analysis and its outcomes, we explored the concept by running TCAD simulations on a representative set of $\mathrm{H}$-shape devices, varying independently the doping density and the mobility. A typical $\mathrm{H}$-shaped device used for TCAD simulations has been also described in Section III and shown in Fig. 3. The following geometrical dimensions, easily achievable with standard CMOS technology, have been taken as reference device throughout this analysis: $L_{\mathrm{NW} 1}=L_{\mathrm{NW} 2}=250 \mathrm{~nm}, L_{\mathrm{C}}=100 \mathrm{~nm}$, $W=50 \mathrm{~nm}$ and $W_{\mathrm{C}}=50 \mathrm{~nm}$. All the regions are made of $n$-type silicon with a constant doping density.

For this study, we intentionally choose three values of doping density and three values of mobility, thus giving 9 possible configurations. Next, the current was extracted on each node with and without the magnetic field (which was set to $1 \mathrm{~T}$ ), and the drain to source voltage was set to $0.1 \mathrm{~V}$.

Results are summarized in Table II. While $I$ and $\Delta I$ depend on the doping and mobility, i.e., on the nanowires resistance, we note that for a given mobility, the ratio $\Delta I / I$ is only a function of the mobility and is almost invariant with the doping. This first "observation" is consistent with the simple predictions from the analytical model (see (28)) and is a major result as it sustains that this approach should be independent of the doping density.

When the mobility changes from 1000 to $250 \mathrm{~cm}^{2}(\mathrm{Vs})^{-1}$, $\Delta I / I$ scales accordingly by varying from about $2.6 \%$ down to $0.6 \%$, independently of the doping level that changes by almost two orders of magnitude, i.e., from $5 \times 10^{17}$ to $1 \times 10^{19} \mathrm{~cm}^{-3}$. Note that for doping densities below $5 \times 10^{17} \mathrm{~cm}^{-3}$ some carrier depletion takes place at the channel interface, thus introducing some "extrinsic" error.

The sensitivity of the device can be improved adding in parallel more interconnecting nanowires similar to NWc. TCAD simulations show that the relative current variation saturates at approximately $3.5 \%$ for a mobility of $1000 \mathrm{~cm}^{2}(\mathrm{Vs})^{-1}$, no matter how many more elements are added to the chain, as compared to $2.6 \%$ for a single element. Therefore, this option is not necessarily attractive, given that all nanowires should also be identical, which is an additional constrain. 


\section{Impact of geometrical mismatch}

Any semiconductor device presents geometrical irregularities, especially when fabricated in a top-down approach. For instance, nanowires may not have the same width and length (line edge/width roughness can lay in the nanometer range) which introduces mismatch in the measurement. More precisely, $\Delta I$ may also contain some inherent geometrical mismatch arising from the length and width of NW1 and NW2. To circumvent this drawback, we propose to consider $\Delta I$ as the additional current generated by the magnetic field only, when varying the length and the width of one of the NW by $5 \%$. Our findings show that this causes less than $4 \%$ deviation of $\Delta I / I$ with respect to a ideal symmetric device. The conclusion is valid also when the conductivity varies, in the "access branches". In fact, all the properties of NW1 and NW2 can be modeled in the circuit model by the resistance $R$.

Additionally, as an effect of the fabrication process, the device may have rounded corners in the regions where N1 and NW2 connect with NWc. We verified the impact of the rounded corners, finding that smaller is the $L_{\mathrm{C}} / W_{\mathrm{C}}$ ratio, higher is mismatch from the TCAD results. For an corner having the radius $25 \mathrm{~nm}$, the value given by the model departs less then $10 \%$ from the ideal case when the radius is 0 . Therefore, we find that the method is also robust in regard to mismatch issues.

In case of low mobility, small dimensions devices, $\Delta I / I$ could decrease drastically making it difficult to measure. However, currents of the order of hundreds of $\mathrm{pA}$ are detectable and can be distinguished from noise with basic equipment for electrical analysis of semiconductor devices [14].

\section{E. Quantitative assessment of the model}

Although the analytical approach aimed at giving the basis for the current splitting approach, we evaluated its accuracy by comparing its predictions with TCAD simulations. Additionally, we introduced an empirical correction to the model. We found that using $0.75 W+3 L_{\mathrm{C}}$ instead of $2 W+L_{\mathrm{C}}$ in (29) improves the model accuracy. Practically, the correction consists in giving a larger weight to the resistance of the NWc, as function of its physical dimensions, in the electrical circuit model that we used. This is consistent with the assumption made when defining $V_{\mathrm{H}}$ in (22). With all the other parameters unchanged, we have now:

$$
\mu=\frac{\frac{\Delta I}{I_{\mathrm{S} 1 / \mathrm{D} 1}}}{r_{\mathrm{H}} B\left(2 \mp \frac{\Delta I}{I_{\mathrm{S} 1 / \mathrm{D} 1}}\right)}\left(\frac{L}{W}+\frac{0.75 W+3 L_{\mathrm{C}}}{W_{\mathrm{C}}}\right) .
$$

Fig. 7 shows the dependence of $\Delta I / I$ on the dimensions of central nanowire NWc (a. and b.) and on the "access regions" NW1 and NW2. Showing also the results of TCAD simulations, these graphs highlight the limitations of the proposed model, (29), and the improvement brought by empirical correction, (30). In a. and b., the dimensions of the central nanowire are varying $\left(W_{\mathrm{C}}\right.$ and $\left.L_{\mathrm{C}}\right)$, whereas in c. and $\mathrm{d}$. the access regions are tuned $\left(W\right.$ and $\left.L_{\mathrm{NW} 1}=2 L\right)$. One
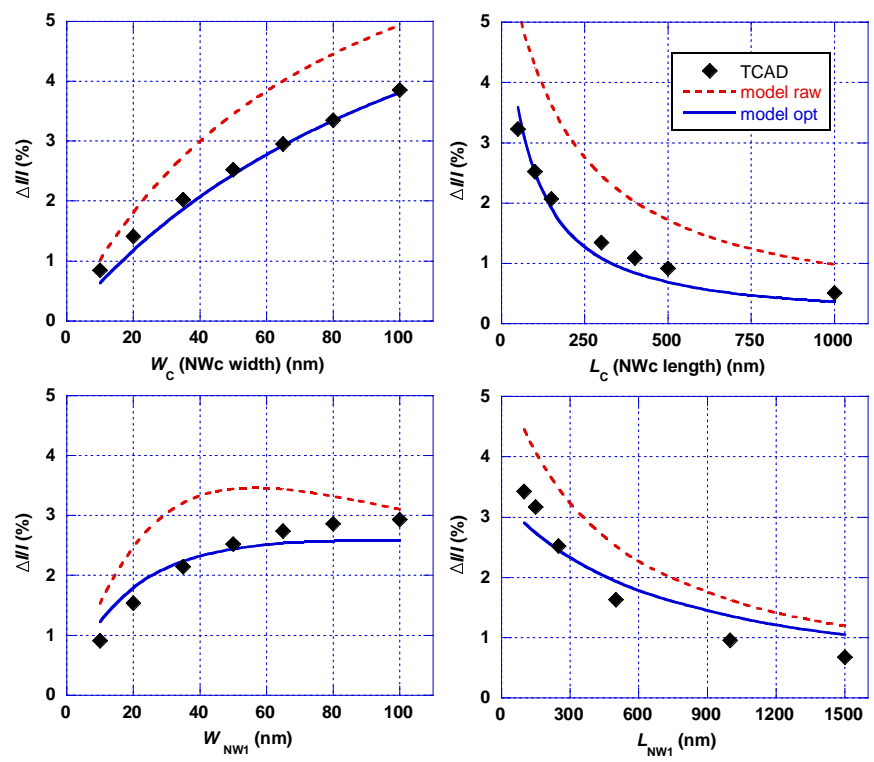

Fig. 7. Impact of various geometric dimensions on the split current sensibility. a. Varying $W_{\mathrm{C}}$, b. varying $L_{\mathrm{C}}$, c. varying $W$, and d. varying $L$. When not varied, the geometric parameters are $L_{\mathrm{NW} 1}=250 \mathrm{~nm}$ (NW1 and NW2 length), $W=50 \mathrm{~nm}, L_{\mathrm{C}}=100 \mathrm{~nm}$ and $W_{\mathrm{C}}=50 \mathrm{~nm} . N_{\mathrm{D}}=1 \times 10^{19} \mathrm{~cm}^{-3}$, $V_{\mathrm{DS}}=0.1 \mathrm{~V}, r_{\mathrm{H}} B=1.15 \mathrm{~T}, \mu=1000 \mathrm{~cm}^{2}(\mathrm{Vs})^{-1}$.

could notice that, although (29) suffers from some inaccuracy (dashed red curves), the trends are fairly well predicted, given the simplicity of the model. On the other hand, using (30) (blue curves) we can have a much better agreement with the TCAD simulation. The model departs from the simulation by less than $15 \%$ for a length $L_{\mathrm{NW} 1}$ between $100 \mathrm{~nm}$ and 500 $\mathrm{nm}$ and for a width $W$ between 20 and $80 \mathrm{~nm}$.

Therefore, (30) seems accurate enough for a first estimation of the mobility in a given topology. Moreover, this formula can also be used to select the most relevant geometrical parameters for a technology node before further refinements obtained from numerical simulations.

\section{DESIGN SPACE AND ABACUS OF H-Shape NWS}

Following the general conclusion brought by both TCAD simulation and the analytical derivation, we will make use of the invariant rules to generate some abacus where the mobility can be directly extracted from the relative change in the current in an H-shape NW topology.

For this purpose, we simulated a series of devices having various dimensions and we plotted the link between the mobility and the ratio $\Delta I / I$ in Fig. 8. Starting from a device having $W_{\mathrm{C}}=W=10 \mathrm{~nm}, L_{\mathrm{C}}=20 \mathrm{~nm}$, and $L=2 L_{\mathrm{C}}=40$ $\mathrm{nm}$, two analysis have been done. In one case, all dimensions have been scaled up by a factor of 2,5 , or 10 ; in the second case only the $L_{\mathrm{C}} / W_{\mathrm{C}}$ ratio was varied from 2 to 3,5 , and 10. The continuous line represents the model as in (27,) the symbols are from TCAD simulations and the dotted lines are linear interpolations of the TCAD simulated points.

Each of the four groups of data corresponds to a distinct $L_{\mathrm{C}} / W_{\mathrm{C}}$ ratio, namely $2,3,5$ and 10 . For each ratio, the different symbols correspond to different dimensions of the nanowire under evaluation (NWc), where the entire device 


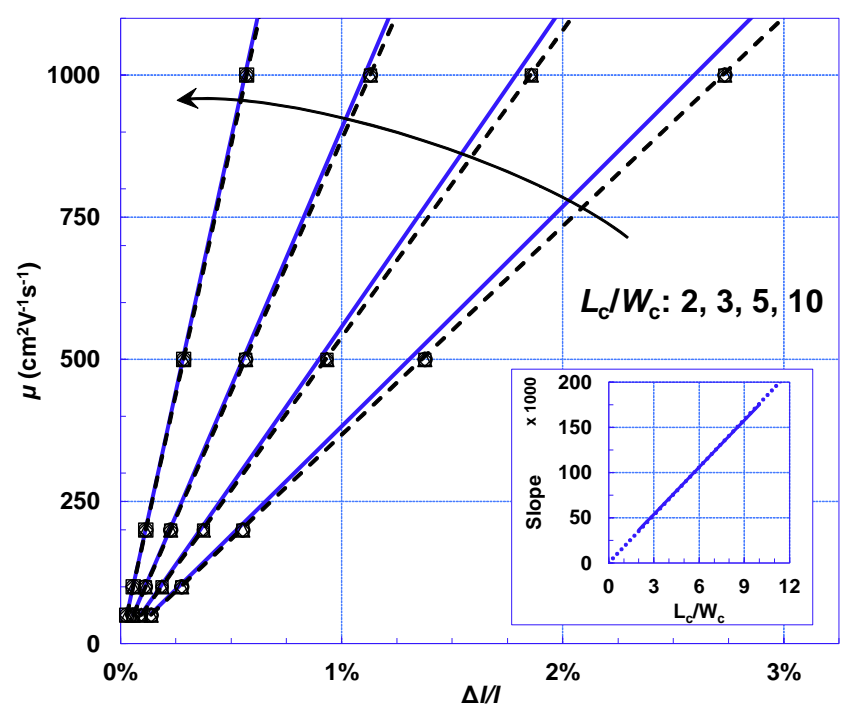

Fig. 8. Guidelines for mobility extraction, as function of the ratio $\Delta I / I$ for an H-shape device. Single marks represent TCAD simulation, dashed lines are their interpolation, and continuous lines are given by (30). From left to right $L_{\mathrm{C}} / W_{\mathrm{C}}$ is $10,5,3$, and 2. Marks in the same points correspond to dimensions of the silicon $\mathrm{H}$-shape device scaled-up uniformly by a factor $1,2,5$, and 10 respectively. $N_{\mathrm{D}}=1 \times 10^{19} \mathrm{~cm}^{-3}, V_{\mathrm{DS}}=0.1 \mathrm{~V}, r_{\mathrm{H}} B=1.15 \mathrm{~T}$.

(therefore including the regions NW1 and NW2 comprised) has also been scaled accordingly, i.e., by a factor of 2, 5 and 10. Therefore, having a given nanowire width, one can use this kind of representation to link $\Delta I / I$ with the mobility, provided that the nanowire length and the access nodes are scaled with respect to some "master" H-shape geometry used to build the abacus. While for $L_{\mathrm{C}} / W_{\mathrm{C}}=2$ the mismatch $\mathrm{TCAD} /$ model is approx. $5 \%$, if $L_{\mathrm{C}} / W_{\mathrm{C}}>3$ the two sets of data almost superpose, thus the model is more precise for higher $L_{\mathrm{C}} / W_{\mathrm{C}}$ ratio. Nevertheless, it might become difficult to extract the $\mu$ when $L_{\mathrm{C}} / W_{\mathrm{C}}$ is higher than 10 . The limitation would come from a practical point of view, given that $\Delta I / I$ might decrease lower than the detectable limits of the measuring equipment. Regarding the global scaling solution, it is interesting to estimate the change of the slope $\mu /(\Delta I / I)$, when the ration $L_{\mathrm{C}} / W_{\mathrm{C}}$ is changed continuously. This is shown in the inset in Fig. 8, which represents a trend line obtained by interpolating the slopes extracted from the four ratios $L_{\mathrm{C}} / W_{\mathrm{C}}$ in the main graph of the same figure. This additional plot generalizes the semi-empirical approach presented in this work, linking the relative current variation to the mobility for any nanowire, provided the $\mathrm{H}$-device follows the predefined design rules.

\section{CONClusion}

From the analysis carried out in this work, we find that the current splitting technique is an interesting alternative to common techniques to extract the mobility in extremely downscaled devices, where the standard Hall effect may suffer from technological and inherent limitations. We have demonstrated the simplicity of this principle on an $\mathrm{H}$-shape nanowire device and developed an analytical model that can be used as a first step towards mobility measurements. Additionally, we presented some interesting invariant laws that make the technique generic and versatile enough with respect to some geometrical variations of the device.

\section{REFERENCES}

[1] G. Ghibaudo, "New method for the extraction of MOSFET parameters," Electronics Letters, vol. 2, no. 9, pp. 543-545, 1988.

[2] J.-P. Colinge, C.-W. Lee, A. Afzalian, N. D. Akhavan, R. Yan, I. Ferain, P. Razavi, B. O'Neill, A. Blake, M. White, A.-M. Kelleher, B. McCarthy, and R. Murphy, "Nanowire transistors without junctions," Nature nanotechnology, vol. 15, pp. 1-5, Feb. 2010.

[3] M. Najmzadeh, J.-M. Sallese, M. Berthome, W. Grabinski, and A. M. Ionescu, "Mobility extraction assessment in GAA Si NW JL FETs with cross-section down to $5 \mathrm{~nm}$," in International Conference on Ultimate Integration on Silicon (ULIS). IEEE, Mar. 2013, pp. 105-108.

[4] J. Chen, T. Saraya, and T. Hiramoto, "Electron mobility in multiple silicon nanowires GAA nMOSFETs on (110) and (100) SOI at room and low temperature," in IEEE International Electron Devices Meeting, no. 110 . IEEE, Dec. 2008, pp. $1-4$.

[5] O. Gunawan, L. Sekaric, and A. Majumdar, "Measurement of carrier mobility in silicon nanowires," Nano letters, vol. 8, no. 6, pp. 1566-71, Jun. 2008

[6] E. C. Garnett, Y.-C. Tseng, D. R. Khanal, J. Wu, J. Bokor, and P. Yang, "Dopant profiling and surface analysis of silicon nanowires using capacitance-voltage measurements," Nature nanotechnology, vol. 4, no. 5, pp. 311-4, May 2009.

[7] J. Chen, T. Saraya, and T. Hiramoto, "Hole Mobility Characteristics in Si Nanowire pMOSFETs on (110) Silicon-on-Insulator," Electron Device Letters, IEEE, vol. 31, no. 11, pp. 1181-1183, 2010.

[8] R. S. Popovic, Hall Effect Devices, 2nd ed. Taylor \& Francis, 2004.

[9] C. Blömers, T. Grap, M. I. Lepsa, J. Moers, S. Trellenkamp, D. Grützmacher, H. Lüth, and T. Schäpers, "Hall effect measurements on InAs nanowires," Applied Physics Letters, vol. 101, no. 15, p. 152106, 2012.

[10] K. Storm, F. Halvardsson, M. Heurlin, D. Lindgren, A. Gustafsson, P. M. Wu, B. Monemar, and L. Samuelson, "Spatially resolved Hall effect measurement in a single semiconductor nanowire." Nature nanotechnology, vol. 7, no. 11, pp. 718-22, Nov. 2012.

[11] J. P. Degrave, D. Liang, and S. Jin, "A General Method to Measure Hall Effect in Nanowires: Examples of FeS2 and MnSi." Nano letters, May 2013.

[12] Synopsys, TCAD Sentaurus Device User Guide. Synopsys, 2012.

[13] J. A. del Alamo and R. M. Swanson, "Measurement of Hall scattering factor in phosphorus-doped silicon," Journal of Applied Physics, vol. 57 , no. 6 , p. 2314,1985 .

[14] I. Keithley Instruments, Low Level Measurements Handbook, 6th ed. Keithley Instruments, 2004.

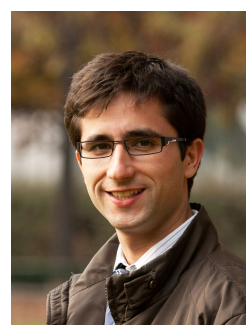

Lucian Barbut received his M.Sc. degree in micro and nanotechnologies for integrated systems from École Polytechnique Fédérale de Lausanne (EPFL), Switzerland; the National Polytechnic Institute of Grenoble, France; and the Polytechnic University of Turin, Italy, in 2010. He is currently working towards the Ph.D. degree with the Electronic Laboratory, EPFL Lausanne, Switzerland. His current research interests include design and fabrication of nanoelectronic devices.

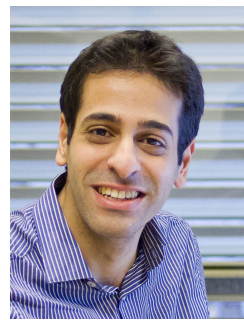

Farzan Jazaeri received his M.Sc. degree in Electronics Engineering (Circuits and Systems Engineering) in 2009 from University of Tehran, Iran. Since December 2011, he has been working toward his $\mathrm{Ph} . \mathrm{D}$. degree in Microsystems and Microelectronics department, at École Polytechnique Fédérale de Lausanne (EPFL), Switzerland.

His research activities involve modeling solid state electron devices. He is currently working on modeling of Junctionless multiple gate devices. 


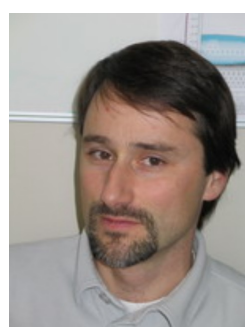

Didier Bouvet received the M.Sc. degree in microelectronic engineering from the University of Lyon, France, in 1991 and the Ph.D. degree in applied physics from École Polytechnique Fédérale de Lausanne (EPFL), Lausanne, Switzerland, in 1997. In 1996, he was engaged as Process Engineer for the startup of ATMEL's 8" fab at Rousset, France. In 1998, he joined the Electronics Laboratories, EPFL, as a Research Engineer and in 2009 he moved to the Center of Micro Nano Technology. His current research interests include design, fabrication, and characterization of sub-micrometer metal-oxide-semiconductor devices and the development of slurries for chemical-mechanical polishing applications.

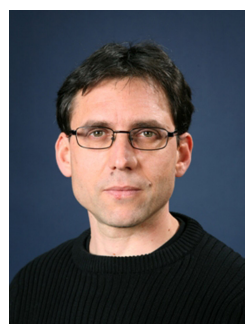

Jean-Michel Sallese received the M.Sc. degree from the Institut National des Sciences Appliquées (France) and the Ph.D in physics from the University/CNRS of Nice-Sophia Antipolis. He is currently Maître d'Enseignement et de Recherche at the Swiss Federal Institute of Technology in Lausanne (EPFL) and his main research interest is on modeling of electron devices. 